\title{
Images and Spectra of the 2017 Total Solar Eclipse Corona From Our Oregon Site
}

\begin{abstract}
Jay M. Pasachoff ${ }^{1 *}$, Christian Lockwood ${ }^{2}$, Erin Meadors ${ }^{2}$, Ross $\mathrm{Yu}^{2}$, Cielo Perez ${ }^{2}$, Marcos A. Peñaloza-Murillo ${ }^{3}$, Daniel B. Seaton ${ }^{4}$, Aris Voulgaris ${ }^{5}$, Ron Dantowitz ${ }^{6}$, Vojtech Rušin ${ }^{7}$ and Thanasis Economou ${ }^{8}$
\end{abstract}

\begin{abstract}
${ }^{1}$ Hopkins Observatory, Williams College, Williamstown, MA, United States, ${ }^{2}$ Astronomy Department, Williams College, Williamstown, MA, United States, ${ }^{3}$ Astronomy Department, Williams College, University of the Andes-Merida, Mérida, Venezuela, ${ }^{4}$ Cooperative Institute for Research in Environmental Sciences, University of Colorado, NOAA National Centers for Environmental Information, Boulder, CO, United States, ${ }^{5}$ Icarus Optomechanics, Thessaloniki, Greece, ${ }^{6}$ Dexter Southfield School, Brookline, MA, United States, ${ }^{7}$ Astronomical Institute, Slovak Academy of Sciences, Tatranská Lomnica, Slovakia,

${ }^{8}$ Enrico Fermi Institute, University of Chicago, Chicago, IL, United States
\end{abstract}

OPEN ACCESS

Edited by:

Scott William McIntosh, National Center for Atmospheric Research (UCAR), United States

Reviewed by:

Harry P. Warren,

United States Naval Research

Laboratory, United States

Bidya Bina Karak,

Indian Institute of Technology (BHU),

India

${ }^{*}$ Correspondence:

Jay M. Pasachoff

eclipse@williams.edu

Specialty section:

This article was submitted to

Stellar and Solar Physics,

a section of the journa

Frontiers in Astronomy and Space

Sciences

Received: 24 July 2018 Accepted: 02 October 2018 Published: 24 October 2018

Citation:

Pasachoff JM, Lockwood C Meadors E, Yu R, Perez C, Peñaloza-Murillo MA, Seaton DB,

Voulgaris A, Dantowitz R, Rušin V and Economou T (2018) Images and Spectra of the 2017 Total Solar Eclipse Corona From Our Oregon Site.

Front. Astron. Space Sci. 5:37. doi: 10.3389/fspas.2018.00037
We report on early results from a suite of instruments for imaging and spectra we deployed to Salem, Oregon, for 2 min of totality at the August 21, 2017, total solar eclipse. Our instruments included refracting telescopes and telephoto lenses for use with CCD detectors and DSLR cameras, narrow-band filters at the wavelengths of coronal emission lines ([Fe XIV] $530.3 \mathrm{~nm}$ and [Fe X] 637.4 nm), and spectrographs. We also monitored the effect of the eclipse penumbra and umbra on the terrestrial atmosphere. The total solar eclipse of August 21, 2017, was the first whose totality crossed only United States territory since the origin of the country, and the first to cross the Continental United States from coast to coast in 99 years. As a result, major campaigns of scientific research and of outreach were carried out.

Keywords: corona, eclipse, sun, spectra, sunspot cycle, saros

\section{INTRODUCTION}

The total solar eclipse of August 21, 2017, was the first whose totality crossed only United States territory since the origin of the country, and the first to cross the Continental United States from coast to coast in 99 years. As a result, major campaigns of scientific research and of outreach were carried out.

Our own expedition team included six scientists, 8 undergraduate students, 3 graduate students, and several collaborators at our main scientific site on the campus of Willamette University, Salem, Oregon. We took advantage of the unusual-for-us ground access by shipping a variety of telescopes, spectrographs, Lyot-type filters, cameras and telescope-mounts across the country by truck in addition to air shipping of international and other equipment. We coordinated with colleagues 65 min farther along the path of totality, in southern Illinois, in order to be able to calculate velocities of changing coronal features.

We were particularly interested in the shape of the corona as part of a synoptic observation set that began early in the previous solar-activity cycle, and linking to older radial-filter observations by Newkirk and others (described with a full set of images in Golub and Pasachoff, 2010, 2014). The ellipticity is greater at solar minimum, and the flattening index in this 2017 eclipse, as well as those measured for the 2019 and 2020 eclipses, should give indications on the strength of the forthcoming cycle, which follows the declining peak of the recently past cycle and its two predecessors. 
Our observations follow earlier high-resolution imaging in streamers (Pasachoff et al., 2007, 2009) and in polar plumes (Pasachoff et al., 2008). We discussed dynamics in Pasachoff et al. (2011) and Pasachoff et al. (2015). We use photographic techniques upgraded from earlier drawings and paintings (Pasachoff and Olson, 2014, 2015).

Pasachoff discussed the range of science carried out at eclipses (Pasachoff, 2009a,b, 2017a,b, 2018a). Pasachoff and Fraknoi (2017) provided a Resource Letter about observing solar eclipses following Pasachoff's Resource Letter about solar science and outreach (Pasachoff, 2010).

\section{OBSERVATIONS AND METHODS}

As is well known, the white light corona-the scattered light of the photosphere on free electrons (Thomson scattering)is a very good indicator of magnetic fields on the Sun, and, these magnetic field lines generate different structures of the solar corona, e.g., helmet streamers, loops, coronal holes, polar plumes, etc., of a different size and brightness. The relationship of the individual white-light coronal structures to the distribution of magnetic fields observed on the surface of the Sun is sometimes quite problematic, particularly, as observed from the coronagraphs on the Solar and Heliospheric Observatory (SOHO) and the Sun Earth Connection Coronal and Heliospheric Investigation (SECCHI; Howard et al., 2008) suite of instruments on NASA's Solar TErrestrial RElations Observatory (STEREO). (The instrument suite was named after Fr. Angelo Secchi, director of the Vatican Observatory in the 19th century, whose 200th birth anniversary was celebrated with a symposium in Rome; Pasachoff, 2018b.) One of the ways to do this is to observe the white-light corona during total solar eclipses at high spatial resolution with subsequent computer data processing, e.g., Koutchmy et al. (1988), Druckmüller et al. (2006). High-quality pictures of the white-light corona during the eclipse and their processing with a computer allows us to distinguish small-scale coronal structures, for example, around 5 arc seconds or less, whose contrast between them is very low, and which are lost with standard exposures. The most recent occasion of this kind was the total solar eclipse observed on August 21, 2017, in a narrow totality path throughout the US, from northwest coast to southeast coast.

We used 20 Nikons, mainly D810s, which lack the moiréreducing filter that has been introduced into many camera models but that slightly degrade the resolution. Our lenses included Nikkor $800 \mathrm{~mm}$ and $400 \mathrm{~mm}$ non-mirror lenses as well as $500 \mathrm{~mm}$ mirror lenses and a Tele Vue refractor. Figure 1 shows a composite made from 11 originals with the $800 \mathrm{~mm}$ lens. Our in-house techniques can now match the quality and contrast accessible in the past for our expedition's and other expeditions' by the high-contrast data reduction made most famous by Druckmüller though now available from several people.

We see the paucity of coronal streamers at high latitudes, revealing the coronal plumes that we studied at the 2006, 2008, and 2009 eclipses (Pasachoff et al., 2007, 2008, 2009, 2011).

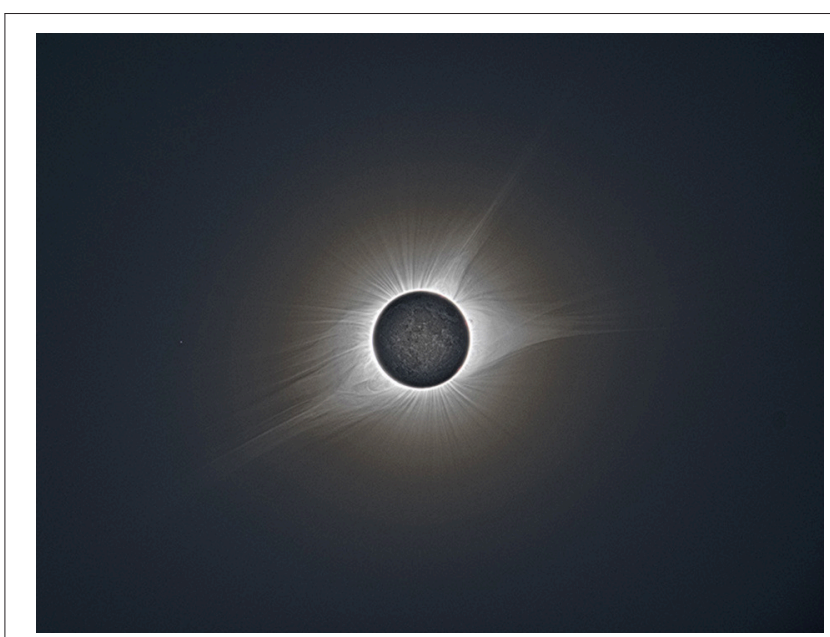

FIGURE 1 | High-contrast imaging showing the paucity of equatorial coronal streamers at this solar-minimum phase of the sunspot cycle, incorporating 11 individual images taken with a Nikon D-810 DSLR camera and a 800-mm Nikkor lens and processed by Christian Lockwood.

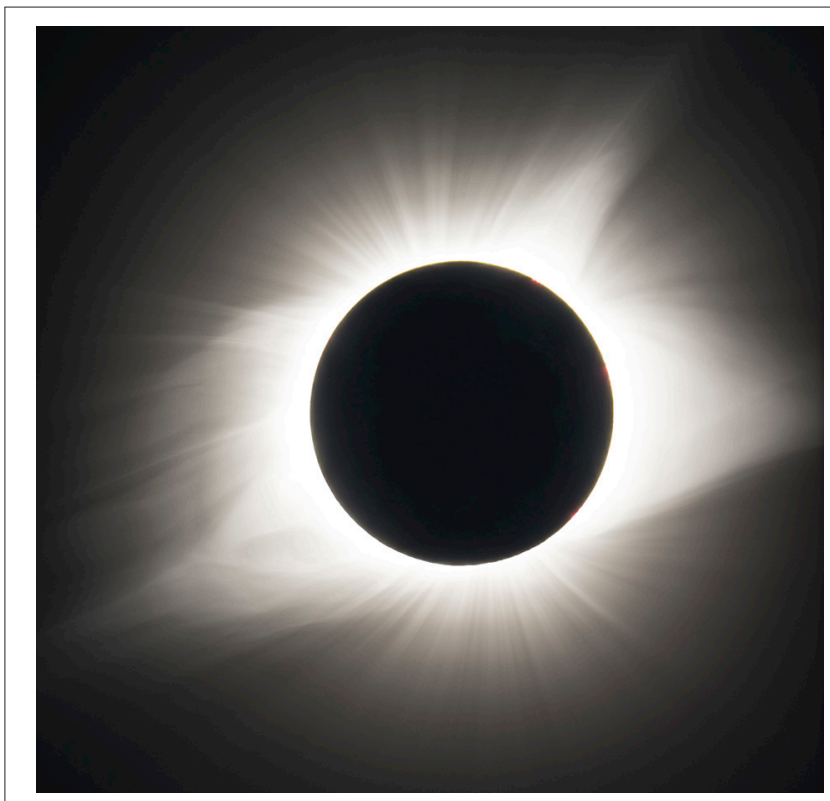

FIGURE 2 | A stack of 3 1/40-s images that is closer to the perception of the eclipse to the unaided eye than the high-contrast composites (Ron Dantowitz)

Figure 2 shows the overall structure of the corona. A composite of our images has been used to successfully compare with the predictions (Mikic et al., 2018) made preeclipse on the basis of the prior month of magnetograms of the solar disk from the HMI instrument on NASA's Solar Dynamics Observatory. Nandy et al. (2018) used a solar-surface flux-transport model for predicting the coronal configuration.

We also obtained a series of flash chromospheric and coronal spectra using high-resolution (HiRes) and low-resolution 
(LoRes) spectrographs. Two slitless spectrographs were used in different dispersion/resolution. These two spectrographs were adapted on an equatorial mount. The LoRes spectrograph was used for the recording of the visual spectrum from $420 \mathrm{~nm}$ up to $680 \mathrm{~nm}$. The second spectrograph (HiRes) was used for the capturing a spectral band from the forbidden [Fe XIV] $530.3 \mathrm{~nm}$ emission line up to the forbidden [Fe X] $637.4 \mathrm{~nm}$ emission line. Our spectrographs correlated with two narrow band Lyot filters tuned to $530 . \mathrm{nm}$ (FWHM $0.38 \mathrm{~nm}$ ) and $637.4 \mathrm{~nm}$ (FWHM $0.5 \mathrm{~nm}$ ) on a $10 \mathrm{~cm}$ Tele Vue refractor with a $220 \mathrm{~mm}$ coelostat.

We brought a meteorological station to measure air temperature, atmospheric pressure, solar radiation, relative humidity and wind (speed plus direction) at our site, to monitor the effect of first the penumbra and then the umbra, continuing the work described in (Peñaloza-Murillo and Pasachoff, 2015). In addition, an array of photo-sensors was used to measure the sky brightness.

\section{RESULTS}

Figure 3 shows a stacked image of the white-light corona as processed by Roman Vanúr from the $800 \mathrm{~mm}$ telephoto lens, using 85 individual pictures of different exposures from 2 to $1 / 500 \mathrm{~s}$. To show higher contrast that reveals the structure of the underlying magnetic field, we used an especially-high-frequency filter. Tian et al. (2017) and Chen et al. (2018) have described the coronal configuration and a coronal cavity over a prominence region, which also shows on our images. Hanaoka et al. (2018) have described changes over an expanse of umbral motion across the United States.

Figure 4 shows a sample slitless spectrum, taken several seconds before $3 \mathrm{rd}$ contact, showing the forbidden [Fe X] coronal red line at $637.4 \mathrm{~nm}$ and the forbidden [Fe XIV] coronal green line at $530.3 \mathrm{~nm}$ as well as chromospheric lines at low heights above the limb. We have been monitoring the ratio of these

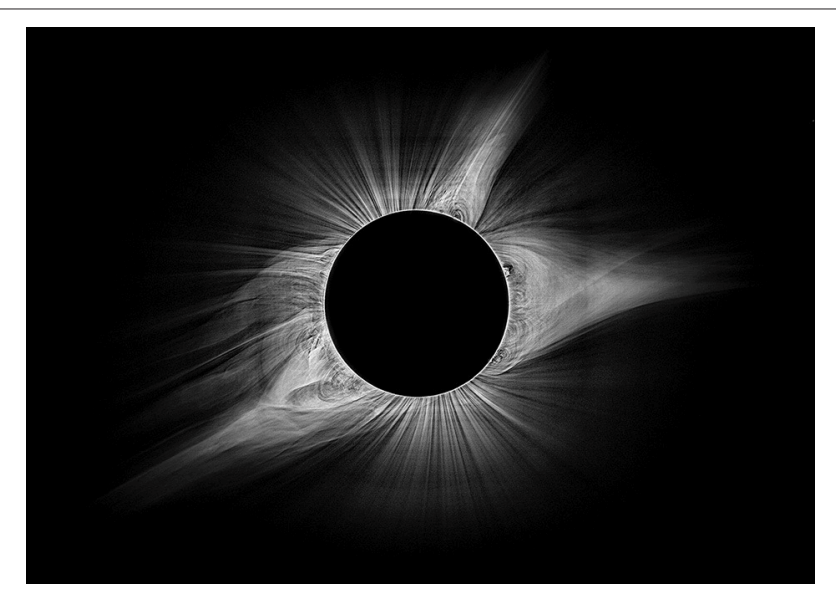

FIGURE 3 | A stack of images taken with an 800-mm f/5.6 lens and displayed at exceedingly high contrast (Jay Pasachoff, Vojtech Rušin, Roman Vanúr, and the Williams College Eclipse Expedition team). spectral lines over the last solar-activity cycle, showing that the lower-ionization line, typical of a coronal temperature of $1 \mathrm{MK}$ or less, is stronger than the higher-ionization line, typical of a coronal temperature of $\sim 1.5 \mathrm{MK}$ at solar minimum, as we found here. In comparison, at solar maximum, we have found the ratio to have been reversed, with the [Fe XIV] line stronger than the [Fe $\mathrm{X}]$ line. Our previous observations include Voulgaris et al. (2010) from the 2006 and 2008 eclipses and Voulgaris et al. (2012) from the 2010 eclipse.

\section{DISCUSSION}

Figure 5 shows the prominent prominence, the innermost corona, and 3rd-contact chromosphere. Our series of observations allowed timing of $1 \mathrm{~m} 54 \mathrm{~s}$ of totality at our site (17:17:21-17:19:15 UTC), which we are comparing with the predictions that depend on the exact value for the solar diameter (Pasachoff et al., 2017d), given the 3-D mapping of the moon in recent years from the Japanese Kaguya spacecraft and the NASA Lunar Reconnaissance Orbiter.

Besides the helmet streamer and threadlike rays, the corona shape was dominated by coronal holes with well-developed polar plumes. The northern coronal hole was located in position angles (PA) $35-338^{\circ}$. There was an interesting intensity break at PA $298-305^{\circ}$ at the solar surface, even though some faint rays were observed in the middle; they expand with height above the limb. This intensity break separated two helmet streamers. The southern coronal hole was located in PA 158-208 ${ }^{\circ}$.

Preliminary comparison of our coronal observations from Oregon with those $65 \mathrm{~min}$ later along the path from Carbondale, Illinois, show a strong, narrow edge of a streamer flipping its orientation, giving a speed of hundreds of $\mathrm{km} \mathrm{s}^{-1}$, as well as a newly brightened polar plume (Pasachoff, 2017c; Pasachoff et al., 2017e, 2018a,b).

After calibrating our spectra, we detected the red coronal line emitting uninterruptedly around the solar limb, even also above the solar magnetic poles. By contrast, the strongest intensity of the green coronal line was detected in two areas, in the east (no sunspots were present) and northeast limbs, caused by the existing-close to the limb-active sunspots 2672 . We have been monitoring the ratio of these spectral lines over the last solaractivity cycle. We detected that the ratio of the intensity of these two lines varies by the area of interest. There are many areas around the solar limb in which the red coronal line is much stronger than green line. The lack of homogeneity in the ratio of two lines means that the coronal temperature strongly varies according from place to place, following the heterogeneity of the two emission lines. The absence of the green emission line signifies a coronal temperature of $1 \mathrm{MK}$ or less. The presence of the green line, the higher-ionization line, is typical of a coronal temperature of $\sim 1.9 \mathrm{MK}$ at solar minimum, as we found over some active areas. In our results, we did not detect the forbidden yellow coronal line of Ca XV at 569.4 nm ( 3-5 MK), even above from the rising sunspots on northeast limb.

We continued our observations of the terrestrial atmosphere and its response to eclipses (Peñaloza-Murillo and Pasachoff, 2015; Pasachoff et al., 2016). The air-temperature measurements 


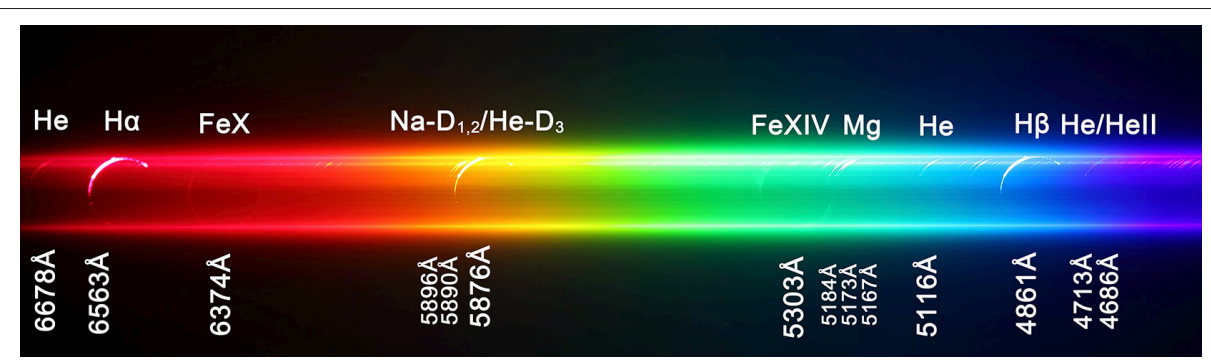

FIGURE 4 | A sample spectrum showing chromospheric lines at 3rd contact as well as forbidden coronal spectral lines, especially the standard [Fe X] at 637.4 nm and [Fe XIV] at $530.3 \mathrm{~nm}$ (Aris Voulgaris).

from our Oregon site on the top of a building on the campus of Willamette University show a drop of $3.1^{\circ} \mathrm{C}=5.5^{\circ} \mathrm{F}$, with a delay of $4.8 \mathrm{~min}$ after central totality, at $0.5 \mathrm{~m}$ above the station base. Other results show that at $1 \mathrm{~m}$ and $2 \mathrm{~m}$ above the station base the drops were $2.5^{\circ} \mathrm{C}=4.5^{\circ} \mathrm{F}$ with a delay of $4.8 \mathrm{~min}$, and $1.6^{\circ} \mathrm{C}=2.9^{\circ} \mathrm{F}$ with a delay of $6 \mathrm{~min}$, respectively. These results were typical for both variables according to those published by Burt (2018) for this eclipse in sites like Whitman (Nebraska), Crossville (Tennessee), and McClellanville (South Carolina). We also detected a slight increase in atmospheric pressure at totality and a pronounced drop in wind speed along with a reduction (symmetric) in variability during the eclipse in accordance with Aplin and Harrison (2003) for the eclipse of August 11, 1999, in the UK. This drop of wind speed is associated with a stabilization in wind direction between $264^{\circ}$ and $312^{\circ}$ clockwise from north.

Figure 6 shows a comparison in which one of our processed eclipse images fills the gap between the on-disk image from the Solar Ultraviolet Imager (SUVI; Seaton and Darnel, 2018) on NOAA's Geostationary Operational Environmental Satellite-16 (GOES-16) spacecraft and a coronagraph image from the Large Angle Spectrometric Coronagraph (LASCO; Brueckner et al., 1995) on board SOHO. The combination of these images enables features to be traced from the disk through our image of the lower and middle corona to the lower-resolution C2 LASCO image.

The basis for our analysis of the large-scale white light coronal structure is this combined image of the eclipse white-light corona, the SOHO/LASCO C2 corona and the GOES-16/SUVI Sun of $30.4 \mathrm{~nm}$. Looking at the SOHO white-light corona, we see three significant and bright helmet streamers, two of which are localized above the western limb of the Sun and one above the southeastern limb of the Sun. Streamers/rays that are less pronounced are located above the eastern limb (three examples) and above the western edge (one example). Similar polar rays also show, though they had been hidden for much of the past decade by high-latitude streamers typical of solar maximum. Pasachoff et al. (2008) had reported on motions and brightness changes in polar plumes during the 2006 total eclipse.

In sum, the white-light corona on August 21, 2017, was observed on the descending branch of cycle 24, whose magnitude according to many features of solar activity-e.g., sunspot numbers, the $2,800 \mathrm{MHz}$ radio flux, the green-line corona intensities, etc.-was the lowest in the last four solar cycles.

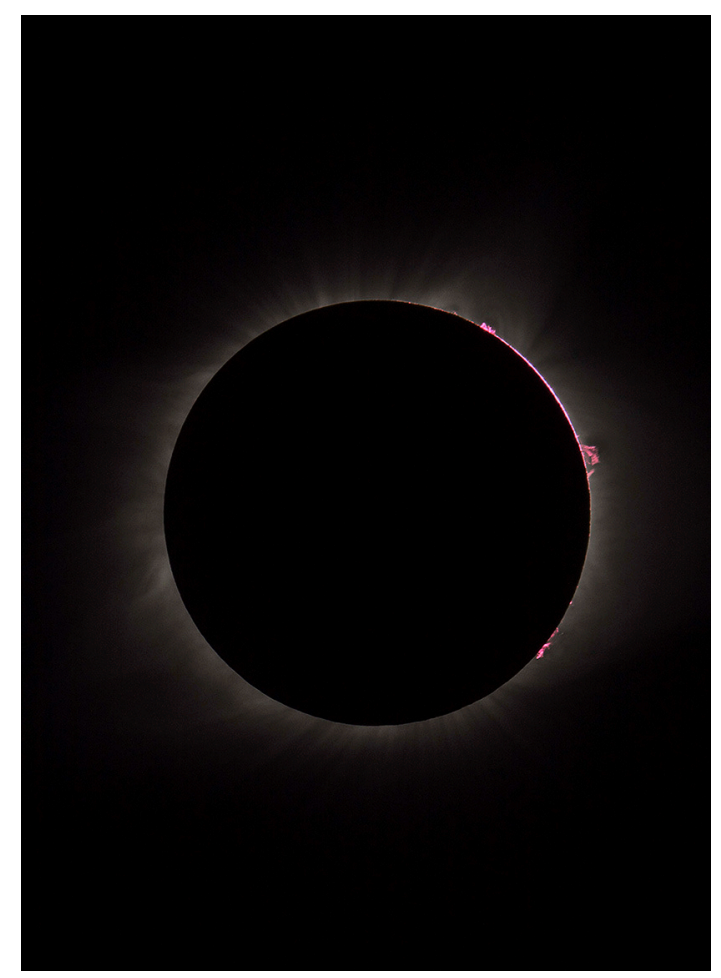

FIGURE 5 | The chromosphere, the innermost corona, and prominences at 3rd contact. Coronal cavities are nicely seen above prominences on the west solar limb (Ron Dantowitz).

Although the solar activity was relatively low through the first part of the year 2017, it increased in August and September. Then the activity again diminished. On the day of the eclipse, the large active area was located in the middle of the solar disk, so it could not be responsible for coronal structures that are observed above the solar limb. However, there was a small sunspot group near the limb on the far side, and became visible the next day as it rotated onto the near side of the disk. Based on our analysis, we come to the following conclusions:

- The shape of the white light corona was a transient type with three or four distinctive helmet streamers and well-developed coronal holes above the two poles of the Sun, where polar plumes were observed. 


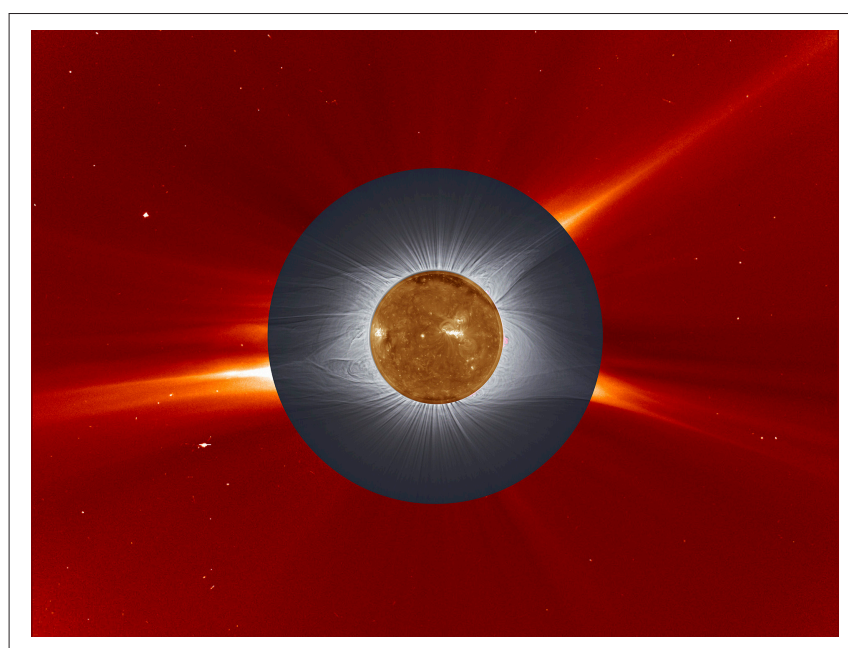

FIGURE 6 | A combination of one of our composite eclipse images between an EUV image from GOES-16/SUVI at $19.5 \mathrm{~nm}$ (corresponding to Fe XI/XII emission at about 1.5 MK) and an image from the LASCO C2 coronagraph, occulting up to 2.5 solar radii revealing the connection between structures in the extended corona and the source regions on the solar disk. It is improved from our Astronomy Picture of the Day image from September 27, 2017 (https://apod.nasa.gov/apod/ap170927.html), with a more detailed eclipse composite and a GOES-16 image instead of the earlier Solar Dynamics Observatory image (eclipse: Jay Pasachoff, Vojtech Rušin, Roman Vanúr, and the Williams College Eclipse Expedition team).

- At the preliminary phase 0.8 of Cycle 24, the Ludendorff flattening index reached 0.24 and replicates the cyclical variability as obtained from many observations since 1851 . In other words, the shape of the white-light corona matched that of similar phase in the previous cycles, in spite of the fact that the peak of this cycle was much lower. (See, for example, http://sidc.oma.be/silso.)

- Just over $1 \mathrm{~h}$ following our observations in Oregon, structural changes occurred in the base of the SE helmet streamer, as well as a change in the brightness of some polar plumes, perhaps showing a change in the flow of particles into the solar wind.

- Above the southeast limb of the Sun, at a height of about 0.85 radius, a dark arc was observed (there are bright arches as well, but the darker is better visible), whose feet at the solar surface are $104^{\circ}$ apart, which is an extraordinary value. Loops or arcs are generally seen in helmet-streamer boundaries, which was also the case with all the helmet streamers.

- Thin coronal rays or loops began to appear in the tops of some of the prominences.

STEREO observations show that the streamers are composed of still-finer-resolution features (DeForest et al., 2018), and our highest quality data reduction matches that idea. High-quality observations of the white-light solar corona, with its processing, allow us to separate different coronal structures (in the sense of their brightness, size, shape, along the line of sight, etc.). Thus, the corona becomes highly structural in every direction, composed of many structures of small dimensions. The white-light corona is also highly dynamic. The coronal structures and their dynamics are linked to the magnetic field, both local and global.
Given the descent into the delayed solar-activity minimum and the desirability of predicting and eventually determining the height and activity of the next solar maximum, we look forward to carrying out similar observations at the total solar eclipses of 2 July 2019 from Chile and of 14 December 2020 from the Atlantic Coast of Argentina. See http://eclipses.info for links and http://totalsolareclipse.org for additional images and references from not only the 2017 total solar eclipse but also from the three partial solar eclipses of 2018.

\section{DATA AVAILABILITY STATEMENT}

The raw data supporting the conclusions of this manuscript will be made available by the authors, without undue reservation, to any qualified researcher.

\section{AUTHOR CONTRIBUTIONS}

JP organized the effort and supplied scientific and logistic backing. CL operated cameras and composited images. EM operated and organized cameras and worked with data. RY organized and operated the meteorological station. CP programmed cameras, operated them, and worked with spectral data. MP-M consulted on the meteorological station and analyzed the data. DS provided the liaisons to space EUV observations. AV built and operated imaging and spectral instruments. RD built and operated imaging and spectral instruments. VR participated in the observing and provided analysis of the images. TE provided the observations from along the totality path.

\section{ACKNOWLEDGMENTS}

The Williams College Eclipse Expedition was supported largely by grants AGS-1602461 from the Solar Terrestrial Program of the Atmospheric and Geospace Sciences Division of the US National Science Foundation and 9876-16 from the Committee for Research and Exploration of the National Geographic Society. Additional student support came from the NASA Massachusetts Space Grant, Sigma Xi (the honorary scientific society), the Slovak Academy of Sciences VEGA project 2/0003/16, the University of Pennsylvania, and the Freeman Foote Expeditionary Fund at Williams College. We thank Dr. Roman Vanúr for his work on white-light data processing. We thank Dr. Christophoros Mouratidis, Hellenic Ministry of Education, Research and Religious Affairs, Kos, Greece for his work on the continuum subtraction of the spectra. We thank Stephen Thorsett, Rick Watkins and Honey Wilson of Willamette University for their hospitality. We thank Tom O’Brien, Photo Engineer for National Geographic Magazine of National Geographic Partners, and Jessica Elfadl of the Exploration Technology Lab of the National Geographic Society for the loan of a Nikon camera and extreme Nikkor telephoto. See http://totalsolareclipse.org or http://sites.williams. edu/eclipse/2017-usa. 


\section{REFERENCES}

Aplin, K. L., and Harrison, R. G. (2003). Meteorological effects of the eclipse of 11 August 1999 in cloudy and clear conditions. Proc. R. Soc. Lond. A 459, 353-371. doi: 10.1098/rspa.2002.1042

Brueckner, G. E., Howard, R. A., Koomen, M. J., Korendyke, C. M., Michels, D. J., Moses, J. D., et al. (1995). The large angle spectrometric coronagraph (LASCO). Solar Phys. 162:357. doi: 10.1007/BF00733434

Burt, S. (2018). Meteorological Impacts of the total solar eclipse of 21 August 2017. Weather 73, 90-95. doi: 10.1002/wea.3210

Chen, Y., Tian, H., Su, Y., Qu, Z., Deng, L., Jibben, P. R., et al. (2018). Diagnosing the magnetic field structure of a coronal cavity observed during the 2017 total solar eclipse. Astrophys. J. 856, 21-30. doi: 10.3847/1538-4357/aaaf68

DeForest, C. E., Howard, R. A., Velli, M., Viall, N., and Vourlidas, A. (2018). The highly structured outer solar corona. Astrophys. J. 862:18. doi: $10.3847 / 1538-4357 /$ aac8e3

Druckmüller, M., Rušin, V., and Minarovjech, M. (2006). A new numerical method of total solar eclipse photography processing. Contr. Astron. Obs. Skalnaté Pleso $36,131-148$.

Golub, L., and Pasachoff, J. M. (2010). The Solar Corona, 2nd Edn. Cambridge, UK: Cambridge University Press.

Golub, L., and Pasachoff, J. M. (2014). Nearest Star: The Surprising Science of Our Sun. 2nd Edn. Cambridge, UK: Cambridge University Press.

Hanaoka, Y., Hasuo, R., Hirose, T., Ikeda, A. C., Shibashi, T., Manago, M., et al. (2018). Solar coronal jets extending to high altitudes observed during the 2017 August 21 total eclipse. Astrophys. J. 860:142. doi: 10.3847/1538-4357/aac49b

Howard, R. A., Moses, J. D., Vourlidas, A., Newmark, J. S., Socker, D. G., Plunkett, S. P., et al. (2008). Sun earth connection coronal and heliospheric investigation (SECCHI). Space Sci. Rev. 136:67. doi: 10.1007/s11214-008-9341-4

Koutchmy, O., Koutchmy, S., Nitschelm, C. H., Sýkora, J., and Smartt, R. N. (1988). "Image processing of coronal pictures," in Solar and Stellar Coronal Structure and Dynamics; A Festschrift in Honor of Dr. John, W. Evans, eds R. C. Altrock (National Solar Observatory/Sacramento Peak, Sunspot, N. M.), 256-266.

Mikic, Z., Downs, C., Linker, J. A., Caplan, R. M., Mackay, D., Upton, L., et al. (2018). Predicting the corona for the 21 August 2017 total solar eclipse. Nat. Astron. 1:0190. doi: 10.1038/s41550-018-0562.5

Nandy, D., Bhowmik, P., Yeates, A. R., Panda, S., Tarafder, R., and Dash, S. (2018). The large-scale coronal structure of the 2017 August 21 great american eclipse: an assessment of solar surface flux transport model enabled predictions and observations. Astrophys. J. 853, 712-717. doi: 10.3847/1538-4357/aaaleb

Pasachoff, J., Person, M. J., Dantowitz, R., Lockwood, C. A., Nagle-McNaughton, T., Meadors, E. N., et al. (2018a). "2018, Early science results from the Williams College eclipse expedition," in 231st AAS, January, National Harbor, MD, 220.06 .

Pasachoff, J. M. (2009a). Solar eclipses as an astrophysical laboratory. Nature 459, 789-795. doi: 10.1038/nature07987

Pasachoff, J. M. (2009b). Scientific observations at total solar eclipses. Res. Astron. Astrophys. 9, 613-634. doi: 10.1088/1674-4527/9/6/001

Pasachoff, J. M. (2010). Resource letter SP-1 on solar physics. Am. J. Phys. 78, 890-901. doi: 10.1119/1.3429977

Pasachoff, J. M. (2017a). The great solar eclipse of 2017. Sci. Am. 317, 54-61. doi: 10.1038/scientificamerican0817-54

Pasachoff, J. M. (2017b). Heliophysics at total solar eclipses. Nat. Astron. 1:0190. doi: 10.1038/s41550-017-0190

Pasachoff, J. M. (2017c). First Results from the August 21, 2017, Total Solar Eclipse. Portland, OR: Solar Physics Division of AAS, 208.03.

Pasachoff, J. M. (2018a). Science at the Great American Eclipse. Astron. Geophys. Roy. Astron. Soc. 59, 4.19-4.23. doi: 10.1093/astrogeo/aty191

Pasachoff, J. M. (2018b). "Secchi's Solar Physics," in International Conference on The Legacy of Angelo Secchi SJ 200 Years After His Birth, Rome, September 3-5. Comitato Nazionale per le Celebrazioni del Bicentenario della Nascita di Angelo Secchi c/o Accademia Nazionale delle Scienze detta dei XL (Denver, CO).

Pasachoff, J. M., Dantowitz, R., Rušin, V., Seiradakis, J. H., Voulgaris, A., Seaton, D. B., et al. (2017e). First 2017-Total-Eclipse Results From the Williams College Team. AGU, New Orleans, SH13B-2476.
Pasachoff, J. M., and Fraknoi, A. (2017). Resource letter OSE-1 on observing solar eclipses. Am. J. Phys. 85, 485-494. doi: 10.1119/1.4985062

Pasachoff, J. M., Jubier, X., and Wright, E. T. (2017d). "Syzygy information: lunar limb profiles at total eclipses of the decade," in 49th DPS, Provo, 417.17.

Pasachoff, J. M., and Olson, R. J. M. (2014). Art of the eclipse. Nature 506, 314-315. doi: $10.1038 / 508314 a$

Pasachoff, J. M., and Olson, R. J. M. (2015). The 1918 eclipse mural series by Howard Russell Butler for the American Museum of Natural History and the Hayden Planetarium. in Inspiration of Astronomical Phenomena VIII: City of Stars, insap.org, eds B. P. Abbott (San Francisco, CA: Astronomical Society of the Pacific), Astronomical Society of the Pacific Conference Series 501.

Pasachoff, J. M., Peñaloza-Murillo, M. A., Carter, A. L., and Roman, M. T. (2016). Terrestrial atmospheric responses on Svalbard to the 20 March 2015 Arctic total solar eclipse under extreme conditions. Phil. Trans. Roy. Soc. A 374:20160188. doi: 10.1098/rsta.2016.0188

Pasachoff, J. M., Rušin, V., Druckmüller, M., Aniol, P., Saniga, M., and Minarovjech, M. (2009). The 2008 August 1 eclipse solar-minimum corona unraveled. Astrophys. J. 702, 1297-1308. doi: 10.1088/0004-637X/702/2/1297

Pasachoff, J. M., Rušin, V., Druckmüller, M., Druckmüllerová, H., Bělík, M., Saniga, M., et al. (2008). Polar plume brightening during the 29 March 2006 total eclipse. Astrophys. J. 682, 638-643. doi: 10.1086/588020

Pasachoff, J. M., Rušin, V., Druckmüller, M., and Saniga, M. (2007). Fine structures in the white-light solar corona at the 2006 eclipse. Astrophys. J. 665, 824-829. doi: $10.1086 / 519680$

Pasachoff, J. M., Rušin, V., Saniga, M., Babcock, B. A., Lu, M., Davis, A. B., et al. (2015). Structure and dynamics of the 13/14 November 2012 white-light corona. Astrophys. J. 800, 90-109, doi: 10.1088/0004-637X/800/2/90

Pasachoff, J. M., Rušin, V., Saniga, M., Druckmüllerová, H., and Babcock, B. A. (2011). Structure and dynamics of the 22 July 2009 eclipse white-light corona. Astrophys. J. 742, 29-42. doi: 10.1088/0004-637X/742/1/29

Pasachoff, J. M., Rušin, V., Vanúr, R., Economou, T., Voulgaris, A., Seiradakis, J. H., et al. (2018b). "Configuration of and motions in the solar corona at the 2017 total solar eclipse," in 232nd AAS Meeting (Denver, CO), June, 325.10.

Peñaloza-Murillo, M. A., and Pasachoff, J. M. (2015). Air-cooling mathematical analysis as inferred from the air temperature observation during the 1st total occultation of the Sun of the 21st century in June 21, 2001, at Lusaka, Zambia. J. Atmos. Solar Terrest. Phys. 125-126, 59-77. doi: 10.1016/j.jastp.2015.02.002

Seaton, D. B., and Darnel, J. M. (2018). Observations of an eruptive solar flare in the extended EUV solar corona. ApJL 852:L9. doi: 10.3847/2041-8213/ aaa28e

Tian, H., Qu, Z., Chen, Y., Deng, L., Huang, Z., Li, H., et al. (2017). Observations of the solar corona during the total solar eclipse on 21 August 2017. Earth Planet. Phys. 1, 68-71. doi: 10.26464/epp2017010

Voulgaris, A., Athanasiadis, T. M., Seiradakis, J. H., and Pasachoff, J. M. (2010). A Comparison of the red and green coronal line intensities at the 29 March 2006 and the 1 August 2008 total solar eclipses:considerations of the temperature of the solar corona. Solar Phys. 264, 45-55. doi: 10.1007/s11207-0109575-7

Voulgaris, A., Gaintatzis, P., Seiradakis, J. H., Pasachoff, J. M., and Economou, T. E. (2012). Spectroscopic coronal observations during the total solar eclipse of 11 July 2010. Solar Phys. 278, 187-202. doi: 10.1007/s11207-0129929-4

Conflict of Interest Statement: The authors declare that the research was conducted in the absence of any commercial or financial relationships that could be construed as a potential conflict of interest.

Copyright () 2018 Pasachoff, Lockwood, Meadors, Yu, Perez, Peñaloza-Murillo, Seaton, Voulgaris, Dantowitz, Rušin and Economou. This is an open-access article distributed under the terms of the Creative Commons Attribution License (CC BY). The use, distribution or reproduction in other forums is permitted, provided the original author(s) and the copyright owner(s) are credited and that the original publication in this journal is cited, in accordance with accepted academic practice. No use, distribution or reproduction is permitted which does not comply with these terms. 